\title{
MIDAS
}

Museus e estudos interdisciplinares

$3 \mid 2014$

Varia e dossier temático: "Museos y participación biográfica"

\section{Regina Cohen, Cristiane Duarte, e Alice Brasileiro - Acessibilidade a Museus}

\section{Fátima Alves}

\section{(2)enEdition}

\section{Journals}

Edição electrónica

URL: http://journals.openedition.org/midas/450

DOI: $10.4000 /$ midas. 450

ISSN: 2182-9543

\section{Editora:}

Alice Semedo, Paulo Simões Rodrigues, Pedro Casaleiro, Raquel Henriques da Silva, Ana Carvalho

\section{Refêrencia eletrónica}

Fátima Alves, "Regina Cohen, Cristiane Duarte, e Alice Brasileiro - Acessibilidade a Museus », MIDAS

[Online], 3 | 2014, posto online no dia 12 maio 2014, consultado no dia 22 setembro 2020. URL : http:// journals.openedition.org/midas/450; DOI : https://doi.org/10.4000/midas.450

\section{Este documento foi criado de forma automática no dia 22 setembro 2020.}

\section{c) (1) (ㅇ)}

Midas is licensed under a Creative Commons Attribution-NonCommercial-ShareAlike 3.0 International License 


\title{
Regina Cohen, Cristiane Duarte, e Alice Brasileiro - Acessibilidade a Museus
}

\author{
Fátima Alves
}

\section{REFERÊNCIA}

Cohen, Regina, Cristiane Duarte, e Alice Brasileiro. 2012. Acessibilidade a Museus. Brasília: Ministério da Cultura, Instituto Brasileiro de Museus. Vol. 2, Cadernos Museológicos. 190 páginas, ISBN 978-85-63078-19-3.

«Let's design all things, all the time, for everyone» - Ronald L. Mace

1 Depois de um primeiro volume dedicado à segurança nos museus, o segundo volume da coleção Cadernos Museológicos é sobre a Acessibilidade a Museus ${ }^{1}$. Publicado pelo Instituto Brasileiro dos Museus (IBRAM) o livro aborda o conceito de acessibilidade no sentido de promover um maior acesso à cultura, à semelhança de outros países (p. ex. Austrália, Canadá, EUA, Reino Unido, França, Espanha, Alemanha, Itália, Bélgica, Suécia e Portugal).

2 Ainda que de forma insuficiente, publicações como Vergnas (1992), Mineiro (2004), Ladman et al. (2005) e Teles (2007), Disability Portfolio Guides (The Resource 2003-2004) e Guides Pratiques de l'Acessibilité (Ministère de la Culture et de la Communication 2007-), entre outros, são representativos da evolução feita neste âmbito, defendendo que um espaço inclusivo vai para além da acessibilidade arquitetónica e inclui o acesso à informação e à participação na programação. Assim sendo, estas melhorias não estão apenas orientadas para as pessoas com deficiência, mas para todos (p. ex. crianças, mulheres grávidas, pessoas com língua materna diferente da do país em que se encontram, pessoas idosas, pessoas com doenças diversas). Por isso, ao reduzirmos ou anularmos as barreiras físicas, sensoriais e cognitivas, criando diversas respostas (visitas, atividades e 
outros eventos) e múltiplos formatos informativos, estamos a caminhar para um espaço mais confortável, seguro e autónomo, e, por sua vez, mais sustentável e para todos.

3 As melhorias no âmbito da promoção da acessibilidade passam pela definição de um plano transversal a todas as ações museológicas, cuja implementação deverá ser pensada a curto, médio e longo prazo, e cujas prioridades devem ser estabelecidas consoante o orçamento da instituição bem como os apoios que possam ser obtidos para o efeito. Aspetos como o espaço, os serviços, as exposições, as reservas, as atividades, os eventos, a sensibilização e a formação dos funcionários com e sem deficiência, inclusive o/a diretor/a e responsáveis de equipas e projetos, assim como a comunicação (p. ex. documentos de divulgação, sítios na internet, redes sociais, entre outros) devem estar implicados ativamente neste processo.

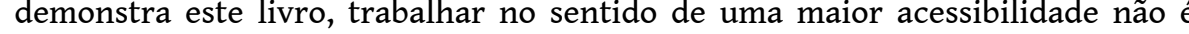
uma tarefa simples, implicando tempo, conhecimento, criatividade e investimento. Por outro lado, é um grande desafio para as equipas, e cujo sucesso implica igualmente o envolvimento de pessoas com deficiência, seja no papel de visitantes, seja como funcionários. Não sendo uma utopia, é um processo de construção, visando alcançar maior qualidade tanto para os cidadãos de hoje como para os de amanhã.

Este caderno museológico pretende oferecer um conjunto de orientações gerais sobre a problemática da acessibilidade e destina-se a um leque diverso de profissionais: técnicos, museólogos, museógrafos, arquitetos, gestores, programadores, entre outros.

o livro configura um manual no qual são apresentadas as temáticas essenciais para enquadrar o tema e é o resultado de vários de anos de trabalho no contexto do Plano Nacional Setorial de Museus brasileiro. A Lei n. ${ }^{2} 11.904$, de 14 de janeiro de 2009, que estabelece a política museológica do país sublinha como sendo os princípios fundamentais dos museus «a universalidade do acesso, o respeito e a valorização à diversidade cultural». Um outro impulso importante para a concretização deste estudo consistiu na realização de um diagnóstico de acessibilidade a todos os museus do Rio de Janeiro, entre 2009 e 2010, o que correspondeu a cerca de $50 \%$ dos museus tutelados pelo IBRAM, através de uma parceria com o Núcleo Pró-Acesso (Núcleo de Pesquisa, Ensino e Projeto sobre Acessibilidade e Desenho Universal) da Faculdade de Arquitetura e Urbanismo da Universidade Federal do Rio de Janeiro.

7 Esta publicação vem confirmar a inclusão da acessibilidade como um tópico fundamental e estratégico na agenda política museológica brasileira, tal como sublinha Mário Chagas num texto introdutório: «A militância a favor da acessibilidade plena parte do reconhecimento de que no cotidiano ela não existe e que, portanto, é preciso construí-la» (xviii). Neste contexto, a promoção da acessibilidade está presente nos documentos fundadores da política atual do IBRAM, sendo assumida como um compromisso no sentido de alcançar um padrão de excelência nesta área (xiii).

o livro organiza-se em torno de seis capítulos principais, acrescendo, no final, um conjunto de anexos referentes a legislação, normas e outra documentação relevante neste domínio.

O primeiro capítulo, «As pessoas com deficiência», oferece uma contextualização sobre algumas terminologias de base acerca da pessoa com deficiência, distinguindo diferentes perceções e categorizações nesta área, e uma panorâmica global de estatísticas sobre a percentagem de pessoas com deficiência no Brasil e no mundo. 
10 No segundo capítulo, «Conceitos básicos», apresenta-se uma definição de acessibilidade e do desenho universal, sendo depois descritos os sete princípios da sua aplicação, e uma definição de rota (percurso) acessível, ou seja, a inexistência de barreiras ao longo de todos os espaços do museu para que possa ser utilizado por uma maior diversidade de pessoas, inclusive com deficiência. Ainda que se reconheça que há situações em que há constrangimentos na realização de adaptações, é sempre possível proporcionar experiências multissensoriais (percorrer, ver, tocar, ouvir e sentir), configurando um desafio fundamental quando se pretende uma maior participação e diversidade de pessoas com e sem deficiência.

«Experiências museais no Brasil e no mundo: ter acesso, percorrer, ver, ouvir, sentir e tocar» corresponde ao terceiro capítulo, no qual são ilustrados vários exemplos de opções inclusivas em alguns museus brasileiros e outros casos no panorama internacional que foram objeto de visita pelas autoras. Porém, gostaríamos de destacar três outros casos de boas práticas neste domínio: a Cité des Sciences et de l'Industrie (Paris), o Science Museum (Boston) e o Victoria and Albert Museum (Londres). A Cité des Sciences et de l'Industrie, criada em 1986, tem uma política de acessibilidade, sendo considerado como um dos museus mais acessíveis do mundo. Quanto ao Science Museum, evidencia-se um longo histórico na melhoria de exposições e de uma constante avaliação desse trabalho; finalmente, o Victoria and Albert Museum, que é um dos casos referenciados pelas autoras, sobretudo em termos da acessibilidade física, é de facto uma instituição com um historial de práticas relevantes na promoção do acesso às exposições permanentes e que tem desenvolvido várias possibilidades de interação, nomeadamente na relação com pessoas cegas e com baixa visão, incluindo experiências em contexto expositivo a nível do toque de objetos. Quanto aos vários museus brasileiros referidos, várias melhorias são assinaladas ao nível do espaço físico, incluindo exemplos de programação adaptada, audioguias e videoguias, e eventos sobre acessibilidade para os públicos com deficiência.

12 Profusamente ilustrado, o quarto capítulo «Acessibilidade a museus: ter acesso, percorrer, ver, ouvir, sentir e tocar» tece um conjunto de recomendações técnicas específicas sobre múltiplos aspetos da acessibilidade, sendo que algumas dessas orientações têm por base um texto publicado em 2004 por duas das autoras, Regina Cohen e Cristiane Duarte, intitulado «Acessibilidade para todos».

13 Por ultimo, "Convivendo com a diversidade», constitui um breve conjunto de considerações sobre o modo como nos devemos relacionar com pessoas com deficiência, desmistificando preconceitos e visando a alteração de atitudes menos respeitosas. Apesar da exaustividade deste caderno, neste contexto importa referir o estudo australiano, Many Voices Making Choices: Museum Audiences with Disabilities (2005), que resume muito bem a forma como devemos encarar e interagir com estes públicos, bem como o que é nesta perspetiva relevante alterar no contexto museal.

De uma maneira geral, este caderno disponibiliza uma lista bastante completa de regras e recomendações para criar condições de acessibilidade, seja ao nível motor, sensorial e cognitivo, alertando para aspetos como a existência de estacionamento próprio, a possibilidade de circulação por todas as áreas do museu, bem como a iluminação dos painéis informativos e objetos, a acústica das salas, a compreensão dos textos e das especificidades dos visitantes cegos, com baixa visão e de surdos.

15 Todavia, neste manual estão ausentes algumas perspetivas, designadamente a acessibilidade dos sítios na internet e a utilização das redes sociais, uma área cada vez 
mais relevante no que concerne à divulgação e comunicação com as pessoas. Muitas vezes, o sítio na internet é o primeiro contacto do potencial visitante com o museu e pode constituir um precioso recurso na procura de informação para uma visita, para esclarecer quanto à adaptação do espaço a pessoas com deficiência e para o acesso virtual às coleções. Assim sendo, este é um aspeto que não deve ser negligenciado na promoção do acesso de forma mais global e integrada. No caso das redes sociais, e em particular o Facebook, são vários os estudos que o apontam como um excelente meio de comunicação junto de todas as pessoas, inclusive das pessoas com deficiência. Refira-se a este propósito experiências bem sucedidas em museus, que através do Facebook desenvolvem uma comunicação especialmente dirigida ao público surdo, utilizando vídeos com recurso à língua gestual.

Por último, embora seja indiscutível a relevância de manuais como este, que, de certo modo, refletem o crescimento de literatura especializada neste campo, note-se que a sua aplicação é ainda de difícil aplicação do ponto de vista dos meios humanos e materiais. Por isso, nesta matéria importa um maior investimento na formação de profissionais de forma a conferir níveis de concretização mais efetivos. Por outro lado, importa integrar estas recomendações na estratégia dos museus ou outras instituições culturais e dar-lhe expressividade também em termos orçamentais. Acreditamos que somente a continuidade destas práticas, a par com uma mudança na postura dos profissionais, poderá trazer um maior sucesso na procura de soluções acessíveis a um maior espectro de públicos. Assim sendo, não basta um conjunto de intenções, mas também colocar em prática a reflexão já existente sobre esta temática, testando $\mathrm{e}$ avaliando... Afinal, o acesso físico, intelectual e cultural é um direito fundamental de todos os cidadãos.

\section{BIBLIOGRAFIA}

Landman, Peta, Kiersten Fishburn, Lynda Kelly, e Susan Tonkin. 2005. Many Voices Making Choices: Museum Audiences with Disabilities. Sidney e Camberra: Australian Museum e National Museum of Australia. http://australianmuseum.net.au/Uploads/Documents/2595/many-voices.pdf

Mineiro, Clara, coord. 2004. Museus e Acessibilidade. Temas de Museologia. Lisboa: Instituto Português de Museus. http://www.ipmuseus.pt/Data/Documents/Recursos/Publica\%C3\%A7oes/ Edicoes_online/Pub_Periodicas/Temas_Museologia/ Temas\%20Museologia_Museus\%20e\%20Acessibilidade.pdf.

Ministère de la Culture et de la Communication. Guides Pratiques de l'Acessibilité. Collection Culture et Handicap [2007-]. Consultado novembro 23, 2013. http://www.culture.gouv.fr/handicap/ guide-intro.html\#2007.

Teles, Paula, ed. 2007. Acessibilidade e Mobilidade para Todos: Apontamentos para uma Melhor Interpretação do DL163/2006 de 8 de agosto. Porto: Secretariado Nacional de Reabilitação e Integração das Pessoas com Deficiência. http://www.inr.pt/uploads/docs/acessibilidade/

GuiaAcessEmobi.pdf 
The Resource (Museums, Libraries \& Archives - MLA). Disability Portfolio Guides [n.ำ1-12; 20032004]. Consultado novembro 23, 2013. http://webarchive.nationalarchives.gov.uk/ 20110802101741/http://www.mla.gov.uk/what/support/toolkits/libraries_disability.

Vergnas, Marie-Laure las, ed. 1992. Des Visites Confortables pour Tous, Cahier des Charges d 'Accessibilité aux Personnes Handicapés. Paris: Cité des Sciences et de l'Industrie.

\section{NOTAS}

1. Além da versão impressa existe uma versão digital da publicação disponível através do seguinte endereço eletrónico: http://www.museus.gov.br/wp-content/uploads/2013/07/ acessibilidade_a_museu_miolo.pdf.

\section{AUTORES}

FÁTIMA ALVES

Acesso Cultura, Portugal, falvezz@gmail.com 